proposition that optimal body temperatures should be evolutionarily conservative may be true but appears to contradict much of the argument made so effectively in earlier chapters; and the support presented is cursory at best. The last section on future directions is disingenuous because its list of "redundant" studies could discourage worthwhile research on the ecology of thermoregulation, and the research 'wish list' is too narrowly focused. There is still a crying need to understand how temperature and its control really affect insects' lives. But a valid point is made: although it is no longer news that insects regulate their body temperature and that this ability affects many other aspects of their biology, some of the physiological mechanisms by which this is done are not so well understood as they might seem.

The Hot-Blooded Insects is an outstand- ing source of information, and can be read with profit and satisfaction by the professional biologist and interested amateur alike. Professionals may find the frequent parenthetical definitions of familiar terms distracting while lay people may wish for more guidance through a few physiological thickets. True initiates in the arcana of insect thermoregulation may be somewhat less pleased, partly because Heinrich presents little new information and batters some pet theory of nearly everyone in the field. Some of the horses he flogs are moribund, if not long dead. On the other hand, he raises many salutary issues on a subject that still has much to contribute to the study of insects and adaptation.

Michael L. May is in the Department of Entomology, Rutgers University, PO Box 231. New Brunswick, New Jersey 08903, USA.

\section{Accidents and complexity}

\author{
H. J. Melosh
}

Solar System Evolution: A New Perspective. By Stuart Ross Taylor. Cambridge University Press: 1993. Pp. 307. £35, $\$ 49.95$.

THIRTY years ago, scientific study of the Solar System was an unpopular backwater of astronomy, shunned by observers who set their sights much farther afield. Speculation about the Solar System's origin was restricted to a few eccentric elder scientists who were not constrained by any real facts about the composition or geological history of the planets. Since then, however, dozens of spacecraft have flown through the Solar System, sending back high-quality images and other data on every planet except Pluto. Samples of rock and soil have been brought back from the Moon, and vastly improved analytical methods have yielded greater insight into the history of meteorites. Planetary science has now blossomed into an active interdisciplinary research area that borrows techniques and ideas from both astronomy and geology.

Many early workers hoped that these findings would quickly reveal a simple comprehensive picture of the Solar System in which such fundamental properties as the densities and compositions of the planets would show readily explainable regularities, and the structure of the Solar System could be seen as an inevitable consequence of the collapse of some kind of protostellar cloud of gas and dust. Instead, the data have revealed a Solar System that is wildly diverse. It seems that stochastic processes have dominated every stage of the Solar System's evolution. Giant impacts of hundreds of planet- sized protoplanets randomly determined the orbits and rotational states of the few survivors. The compositions of the giant planets were established by a hasty, lastminute competition between accretion onto protoplanetary cores and dissipation of the solar nebula by violent outbursts of the young Sun. Even the meteorites, presumed to be slightly altered samples of the most ancient solid bodies in the Solar System, show a numbing complexity that has stubbornly resisted broad generalizations. Although the full implications of the new catastrophic view of Solar System formation have yet to be spelled out, the outlines of the new-world picture are becoming clearer.

Reducing the vast amount of data to manageable proportions is a monumental task. It is easy to get sidetracked on knotty but fascinating problems and lose sight of the overall picture. Stuart Ross Taylor, a geochemist by profession, has taken a broad view of Solar System evolution, and his most recent book is a clear distillation of his work. Although he naturally inclines to geo- or cosmo-chemical arguments, he has not ignored constraints from physics, astronomy and geology, making this the first coherent account of the new view of Solar System history. Although most of the individual facts in the book can be found in one or another of the massive tomes in the University of Arizona Press's Space Science Series (to which Taylor makes repeated references), this book offers a readable and concise introduction.

After a brief historical and philosophical discussion of planetary formation, Taylor describes nebular accretion models, putting the formation of our Solar
System into the context of the Universe and the Galaxy. The discussion of nebular collapse is necessarily vague and will probably be rapidly revised as radioastronomical techniques continue to resolve star formation in nearby molecular clouds. Moving from theory to evidence, he devotes his longest chapter to meteorites, offering a solid, no-nonsense separation of facts from nonfacts and of plausible theories from wishful thinking. Not everyone will agree with his conclusions, but he does discuss the main issues in an unbiased way. In his next chapter, he covers impacts, a testimony to the importance recently attached to impact cratering. Much of the discussion centres on the lunar cratering record, but most of the other principal topics are touched on. He then gives a planet-by-planet description of the main bodies in the Solar System, with emphasis on the geochemical properties of the terrestrial planets and asteroids, although the giant planets are also briefly described. Finally, he discusses rings and satellites while debunking the myth that planetary satellite systems are miniature Solar Systems. As is to be expected of a book fresh from the front lines of scientific research, many of the issues are not yet satisfactorily resolved, such as the origins of chondrules, the age of planetary ring systems, and the nature of heat sources in the early solar nebula; but Taylor gives a balanced discussion of what is known about each of these problems. He ends his book with a philosophical consideration of the complexity of the Solar System.

This is not a book for beginners or those on undergraduate courses. Without some acquaintance with geochemistry and isotopic dating methods readers will be quickly lost. Topics are presented roughly in order of their appearance in the evolving Solar System, a format which often presumes that the reader has knowledge of facts and ideas long before they are introduced in detail. The discussion of meteorites therefore begins with calciumaluminum inclusions, proceeds to chondrules and only later addresses meteorites themselves. This approach demands quite a bit of repetitive discussion of the same topic in different contexts, and often breaks up the presentation. For graduate courses, however, where students have been previously acquainted with planetary science, the book should serve as an excellent reference: it succinctly summarizes evidence and arguments. But its main audience will be professional Earth and planetary scientists or knowledgeable amateurs who want a readable introduction to the latest ideas on the origin and evolution of the Solar System.

H. J. Melosh is in the Lunar and Planetary Laboratory, University of Arizona, Tucson. Arizona 85721, USA. 